\title{
EVALUACIÓN DE IMPACTO DEL PROYECTO DE VINCULACIÓN CON LA COLECTIVIDAD EN EL PROGRAMA DE CAPACITACIÓN CONTABLE FINANCIERA PARA MUJERES EMPRENDEDORAS EN EL CANTÓN CAYAMBE
}

\author{
IMPACT EVALUATION OF THE PROJECT OF LINKING \\ WITH THE COMMUNITY IN THE PROGRAM OF FINANCIAL \\ ACCOUNTING TRAINING FOR WOMEN ENTREPRENEURS \\ IN THE CANTON OF CAYAMBE
}

\author{
Pedro David Rodriguez Salazar', Diego Marcelo Mantilla Garcés², \\ Kerwin Eduardo Zumarraga Marroquin ${ }^{3}$, Marco Vinicio Cevallos Bravo ${ }^{4}$
}

\begin{abstract}
Resumen
Los proyectos de vinculación con la sociedad buscan generar un impacto social positivo en las comunidades, pero, en muchas ocasiones, esto no se analiza posterior a su aplicación. Es por esta razón que el presente estudio analiza dicho impacto de uno de los proyectos de vinculación con la sociedad de la Facultad de Ciencias Administrativas de la Universidad Central del Ecuador, determinando de esta manera, si dicho proyecto fue beneficioso y de ser el caso plantear la posibilidad de continuar y replicarlo. Por medio del presente estudio se plantea un modelo aplicable para la evaluación del impacto social de otros proyectos de vinculación.
\end{abstract}

Palabras clave

Impacto social, proyecto, vinculación con la sociedad.

Abstract

The projects linked to society aim to generate a positive social impact in the communities, but in many circumstances, this is not analyzed after its application, for this reason the present study analyzes the impact of one of the projects linked to society of the Faculty of Administrative Sciences of the Central University of Ecuador, defining in this way if said project was useful, and if in the case, to propose the possibility of continuing and replicating it. Through the present study, an applicable model for evaluating the social impact of other linked projects is proposed.

Keywords

Social impact, project, linkage with society. 


\section{Introducción}

La universidad es una institución esencial del Estado, es la encargada de mantener, con espíritu crítico, los conocimientos científicos actualizados en los diversos campos, y con ellos proveer de las habilidades necesarias en diversas disciplinas para formar profesionales, posgraduados y doctores que el país requiere. La universidad, mediante sus actividades de investigación, docencia y servicios, contribuye a la identidad nacional buscando la óptima calidad de vida, individual y colectiva, expresada por la satisfacción de las necesidades humanas, como derechos, tanto del dominio biológico, como del cultural de la ciencia y el arte, y el social de la producción y el consumo (Hermida, 2019).

En este contexto, la Universidad Central del Ecuador desarrolla su programa de vinculación con la sociedad, con la participación de sus estudiantes, docentes y empleados, aplicando herramientas de desarrollo rural para conocer las necesidades con la propia comunidad; y recoger sus aspiraciones para tratarlas de manera interdisciplinaria en el campo de las ciencias de la vida y el ambiente, de la vida familiar y la vivienda con la participación de sus diferentes facultades que desarrollan una interacción mutua entre la universidad y la comunidad.

Este tipo de programas permiten que se pueda relacionar a la carrera con el ámbito social, contribuyendo con las actividades económicas que permiten el desarrollo de ciertas zonas de nuestro país, por lo que es necesaria la aplicación de los conocimientos tanto de estudiantes como de docentes. Por otra parte, el acercamientos de los alumnos hacia la parte real, como son las personas y los negocios, permite que se aplique la actividad académica en la parte práctica.

\section{Marco teórico y metodológico}

El fomento de la actividad emprendedora cada vez toma más importancia en América Latina y el Caribe. Agrupaciones no gubernamentales locales, hasta entidades públicas de nivel nacional están comenzando a implementar proyectos para difundir la cultura emprendedora y articular la creación de nuevos negocios. El desarrollo de emprendedores y nuevas empresas se ha convertido en un objetivo central para acelerar el progreso económico y social de las naciones (Angelelli y Prats, 2005).

Los ecuatorianos poseen una alta iniciativa orientada al emprendimiento, esta afirmación se sustenta en estadísticas del Global Entrepreneurship Monitor (Gem, por sus siglas en inglés) que ubica a Ecuador con una de las tasas más altas de actividad emprendedora temprana regional $(31,8 \%)$ delante de Colombia Perú y Chile (Lasio, 2016).

Esta primera contextualización podría ser muy alentadora, pero la mayor parte de los emprendedores ecuatorianos se han motivado por necesidad y falta de empleo formal. Esta caracterización determina una cadena de empresas muy pequeñas, con bajo nivel de innovación y concentración preferente en el comercio, lo que conlleva por añadidura a escaza generación de valor agregado (Robayo, 2009).

Robayo (2009) afirma que para mejorar el nivel de emprendimientos en Ecuador hay que fortalecer el ecosistema emprendedor $y$, en esa perspectiva, juega un rol fundamental la academia. De manera pragmática, cuando los estudiantes concluyen su formación profesional y se encuentran con una realidad que limita las posibilidades de acceso a un empleo, se dispara en ellos, como una ráfaga, el emprendimiento generado por la necesidad. 
Una de las variables fundamentales que el Gem analiza para comprender la motivación en distintos tipos de emprendedores es la educación. La percepción del emprendimiento respecto al nivel de educación deja en claro que, mientras más alto es el mismo, la posibilidad de identificar oportunidades de mejora al emprender se incrementa.

Sin embargo, la realidad ecuatoriana evidencia que la educación superior no está aportando al surgimiento de potenciales emprendedores por oportunidad: "En Ecuador predominan los emprendedores que han concluido la educación primaria, 32\%, y la educación secundaria, 33\%. Entre los emprendedores nacientes destaca la proporción con primaria completa y entre los nuevos, aquellos con secundaria completa. La proporción de emprendedores con estudios superiores no alcanza el 15\%." (Lasio, 2016).

En esta perspectiva el rol de los centros educativos resulta de vital importancia en la formación vocacional, son cada vez más frecuentes los concursos de ideas, los espacios para compartir con emprendedores, y el desarrollo de actividades de distinta índole que no solo buscan fomentar la formación sino además la creación de redes y de contactos que son insumos vitales para el éxito del emprendimiento (Ortega y Kamiya, 2013).

\section{Educación contable}

El autor Ángel García (2017) nos menciona: "El componente de ciencias contables y financieras, está orientado a formar al estudiante en los procedimientos y técnicas para el registro y representación de las transacciones económicas [...]. Proporciona la fundamentación teórica y la ejercitación práctica necesaria para el manejo contable y financiero, la comprensión de situaciones de riesgo y la operación de mercados de capitales".

"La contabilidad se presenta la necesidad de interrumpir la llamada 'parálisis pa- radigmática' que afronta la ciencia contable, producto de haberse reducido a la ecuación patrimonial: $A=P+C$, que solo permite cuantificar un equilibrio aparente, porque no toma en cuenta aspectos del entorno, dando como resultado cifras frías sin contexto, basadas en métodos y normas" (Saavedra, 2015).

"En la adquisición de los conocimientos elementales que formarán parte del quehacer diario y que repercutirá en el desempeño laboral, influyen elementos psicológicos y sociológicos que se deben tener en cuenta a la hora de ejecutar un programa de capacitación, las emociones, motivaciones y deseos de los recursos humanos para perfilar su capacitación, son elementos indispensables en el trabajo personalizado" (Herrandiz, 2016).

"Es relevante destacar la influencia de las universidades como entes generadores de conocimiento donde se estudie la mejor opción para implementar planes o programas que incentiven a los individuos a desarrollar, por voluntad propia, de forma responsable y con plenos conocimientos tanto científicos, técnicos como empíricos, emprendimientos sostenibles" (Araque, 2016).

\section{Inclusión productiva}

"Inclusión productiva rural es la condición en la cual las personas y comunidades rurales en condición de pobreza y vulnerabilidad tienen acceso a las oportunidades para trabajar más productivamente, y cuentan con las capacidades y activos suficientes para ello [...]. Habilitar a las personas en condición de pobreza para invertir y para adoptar estrategias con mayor rentabilidad y riesgo" (Berdegué, 2015).

"Las intervenciones del Proyecto Alianzas Productivas son suficientes para propiciar relaciones comerciales duraderas, pero ellas necesitan un período de maduración [...] un hogar beneficiario que lleva más 
de 3 años en el proyecto tiene un ingreso por la venta del producto apoyado por la alianza que es 45,06\% más alto que el del hogar que lleva menos tiempo" (Mark Lundy, 2015).

"La educación técnica de calidad se constituye un elemento clave en la lucha por la superación de la pobreza, la igualdad de oportunidades y la movilidad social. En alguna medida, las brechas en inclusión social, productiva y de pobreza son el resultado de un desacople entre lo que las escuelas enseñan y lo que el mercado laboral requiere, lo que se traduce en escasa competencia profesional con empleos inestables y mal remunerados" (Tomaselli, 2018).

"Establece técnicas de gestión contable y herramientas de control adecuadas a los procesos de toma de decisiones en una entidad de forma creativa, analítica y profunda para adecuarla a las exigencias propias del desarrollo económico local" (Arencibia, 2018).

\section{Impacto económico}

"El conocimiento forjado en las universidades adquiere un rol central en el proceso productivo y competitivo de la economía, debido no solo por la adquisición del conocimiento permanente, sino por la generación y aplicación de nuevas tecnologías traducidas en una habilidad más competitiva" (José Barragán Codina, 2017).

La educación ayuda a edificar la sociedad del futuro, por lo tanto la misma es una inversión en capital humano, que desde un punto de vista económico debería reportar beneficios y de acuerdo a lo social ayudar a formar mejores ciudadanos, y por ende a una mejor sociedad (Cañón Rodríguez, 2016).

\section{Participantes}

Los participantes de la presente investigación fueron las ciudadanos del cantón Cayambe que participantes del proyecto de vinculación con la colectividad de la Facultad de Ciencias Administrativas denominado: Programa de Inclusión Productiva y Educación Contable Financiera para Mujeres Microemprendedoras del Cantón Cayambe, realizado durante el período 2016. Que fueron 60 personas del Mercado Juan Montalvo y 100 personas del Mercado Diario.

\section{Diseño de investigación}

En el presente estudio se utiliza el método cuantitativo para análisis de resultados, una investigación exploratoria de campo con una muestra aleatoria; es decir, todos los integrantes de la población tienen la misma posibilidad de ser seleccionados. El estudio fue aplicado sobre la población que se ha considerado a los 160 participantes del proyecto de vinculación con la colectividad.

\section{Instrumentos y técnicas de recolección de datos}

Las variables utilizadas para el cálculo de la muestra son:

$\mathrm{N}$ : tamaño de la población que está comprendida por 160 personas que participaron en el proyecto de vinculación con la sociedad del cantón Cayambe.

E: el error maestral considerado fue del $5 \%$ a fin de seleccionar una muestra significativa de la población, evitando la necesidad de entrevistar a todos los participantes originales del proyecto de vinculación por la dificultad del proceso.

$$
n=\frac{N}{E^{2}(N-1)+1}
$$

El proyecto de vinculación con la sociedad se lo llevó a cabo con la participación de dos mercados de Cayambe: el Mercado de Juan Montalvo con el 38\% del total de la población; y el Mercado Diario con el 62\%. Por 
esta razón la muestra fue de 115 encuestas, de las cuales se realizaron. 44 en el Mercado Juan Montalvo, y 71 en el Mercado Diario.

\section{Plan de análisis de datos}

En la investigación se realizó un análisis cuantitativo mediante la aplicación del programa SPSS para tratamiento de bases de datos, con la finalidad de realizar cruces de variables para obtener mejores resultados en la investigación. Los datos cualitativos de la investigación fueron organizados mediante el programa Atlas T.I.

\section{Procedimiento}

Para la investigación se realizó una investigación de campo en los mercados mencionados. Las encuestas fueron aleatorias para que los participantes del estudio tengan la misma probabilidad de ser seleccionados $y$, de esta manera, evitar sesgar los resultados en cuanto a información crítica. Por ejemplo: la edad de los encuestados podría incidir en la perspectiva de la calidad de los cursos.

\section{Metodología del BID}

Como metodología para el desarrollo de la investigación, se aplicó la Hoja de Ruta presentada en el libro La evaluación de impacto en la práctica y el manual del encuestador, de la metodología del Banco Interamericano de Desarrollo (BID).

Se revisaron los diversos objetivos que una evaluación de impacto puede lograr y los indicadores de resultados y del tamaño de los efectos anticipados.

El cuestionario de evaluación partió de las medidas de resultado que se utilizarán para evaluar el desempeño y establecer si el programa de vinculación con la sociedad, desarrollado por la Facultad de Ciencias Administrativas de la Universidad Central del Ecuador, tuvo éxito o a futuro se deberá reformular, considerando los indicadores de resultados.

Una evaluación de impacto constituye un tipo particular de evaluación ya que busca responder a una pregunta orientad a identificar una causa y un efecto: ¿Cuál es el impacto (o efecto causal) de un programa y su influencia en los resultados de interés?, centrándose en los cambios directamente atribuibles al programa desarrollado después de un tiempo determinado.

Se utilizó El manual del encuestador aplicado en la metodología del BID como herramienta de direccionamiento pare el levantamiento de la información y se formularon indicadores definidos en la metodología del BID aplicables a la presente investigación, según la siguiente tabla:

Tabla 1. Indicadores de medición de impacto

\begin{tabular}{|c|c|c|c|}
\hline Ìtem & Indicador & Fórmula indicadora & Hipótesis \\
\hline 1 & $\begin{array}{l}\text { Porcentaje alcanzado } \\
\text { en cada uno de los ob- } \\
\text { jetivos del proyecto (ge- } \\
\text { neral y específicos) }\end{array}$ & $\begin{array}{l}\text { No. de beneficiarios que consideran que } \\
\text { se alcanzó el objetivo } \\
\text { No. de beneficiarios del proyecto }\end{array}$ & $\begin{array}{l}\text { H1: Los beneficiarios del proyecto de } \\
\text { vinculación con la sociedad conside- } \\
\text { ran que se cumplió con el objetivo. }\end{array}$ \\
\hline 2 & $\begin{array}{l}\text { Porcentaje de partici- } \\
\text { pantes que mejoraron } \\
\text { su calidad de vida des- } \\
\text { pués de la aplicación del } \\
\text { proyecto de vinculación. }\end{array}$ & $\begin{array}{l}\text { No. de participantes que consideran que } \\
\text { se mejoró su calidad de vida. } \\
\text { No. total de participantes }\end{array}$ & $\begin{array}{l}\text { H1: Los participantes del proyecto de } \\
\text { vinculación con la sociedad mejora- } \\
\text { ron su calidad de vida. }\end{array}$ \\
\hline
\end{tabular}




\section{Análisis de datos}

El proyecto de vinculación realizado estuvo enfocado a las mujeres emprendedoras del cantón Cayambe, por lo que en el presente análisis se realizará una comparación entre diferentes variables importantes para la investigación y que determinen resultados en el estudio.

\section{Variable: edad de participantes}

La edad de las mujeres en el estudio es una variable que se debe analizar en dicho proyecto, ya que esto nos permite identificar entre que rangos se encuentra la mayor parte de participantes y en el momento de cruzar los resultados con otras variables determinar las diferencias en el comportamiento de acuerdo a las edades.

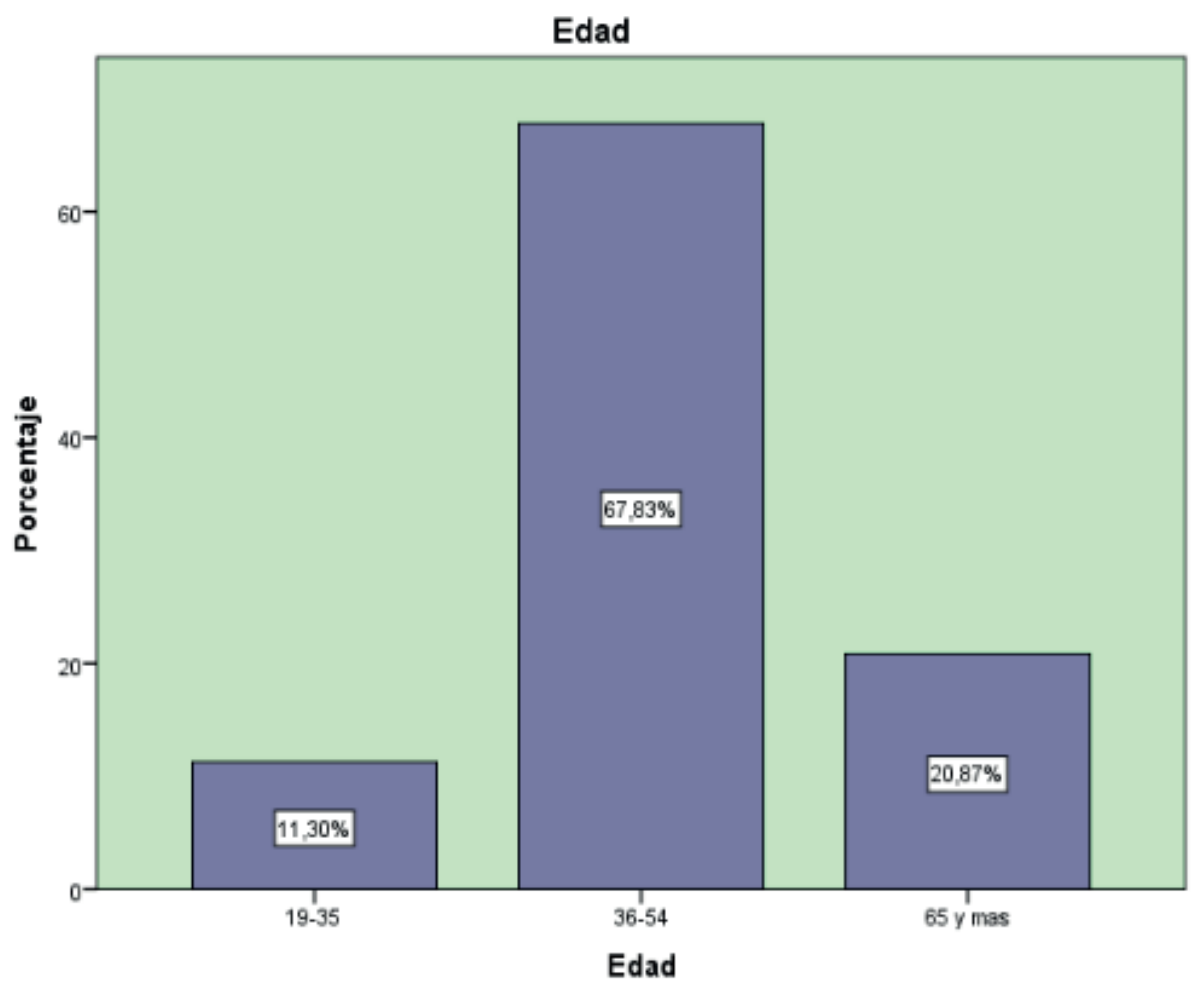

\section{Resultado}

Se determinó que el $11,30 \%$ se encuentra entre 19 y 35 años, el 67,83\% se encuentran entre 36 y 54 años y el 20,87\% tiene más de 65 años. Es decir, la mayor parte de mujeres emprendedoras tienen a partir 36 años, esto permite analizar la metodología con la que se pueda llegar de mejor manera a dicho sector, el tipo de negocio que prefieren y la incidencia que tuvo nuestro programa.

\section{Cariable: tipo de negocio}

Esta variable es muy importante ya que nos permitió determinar en qué ámbito necesitaban mayor capacitación, tomando en cuenta el tipo de negocio que llevaban a cabo, y con ello, hacer una relación con la variable edad, identificando qué tipo de negocio prefieren las mujeres de acuerdo a su rango de edad. 


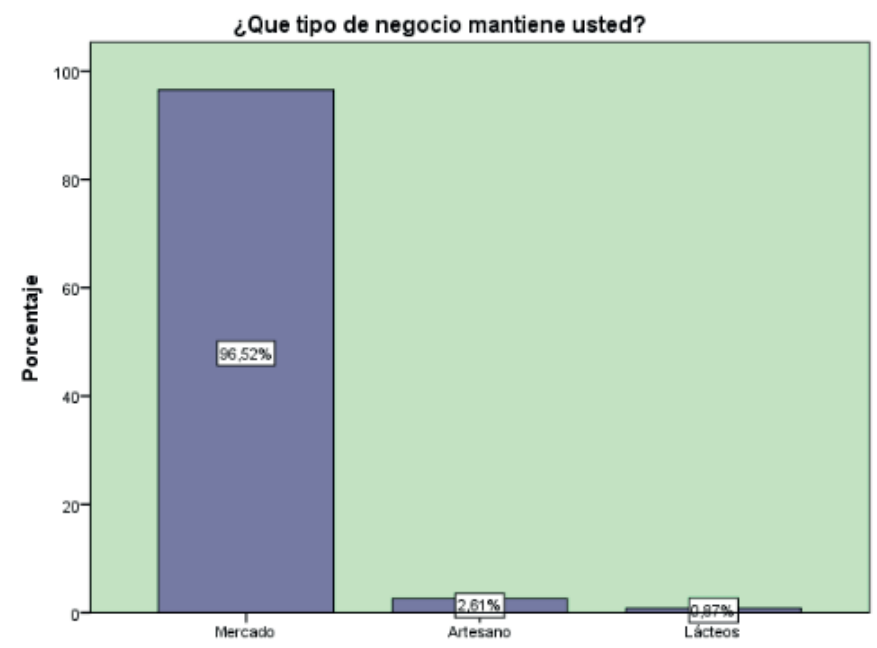

\section{Resultado}

Se obtuvo que el $100 \%$ de los participantes tienen un negocio propio de los cuales el 96.52\% mantiene negocios en el mercado, el $2,61 \%$ son artesanos y el $0,87 \%$ se dedican a la industria láctea; es decir, la mayor parte de mujeres se dedican al negocio que tienen que ver con la cosecha, intermediación y comercialización de productos. Estos resultados muestran la importancia que tuvo el proyecto ya que la contabilidad que deben llevar las mujeres es trascendental para el éxito de sus negocios, así como el manejo de costos y otros aspectos en los que se llevaron a cabo en la capacitación.

\section{Variable: conocimientos}

Esta variable es una de las más importantes en el proceso del proyecto, ya que el impacto que tiene en las participantes determina el éxito de los objetivos planteados, y con ello la continuidad en diferentes sectores de nuestro país.

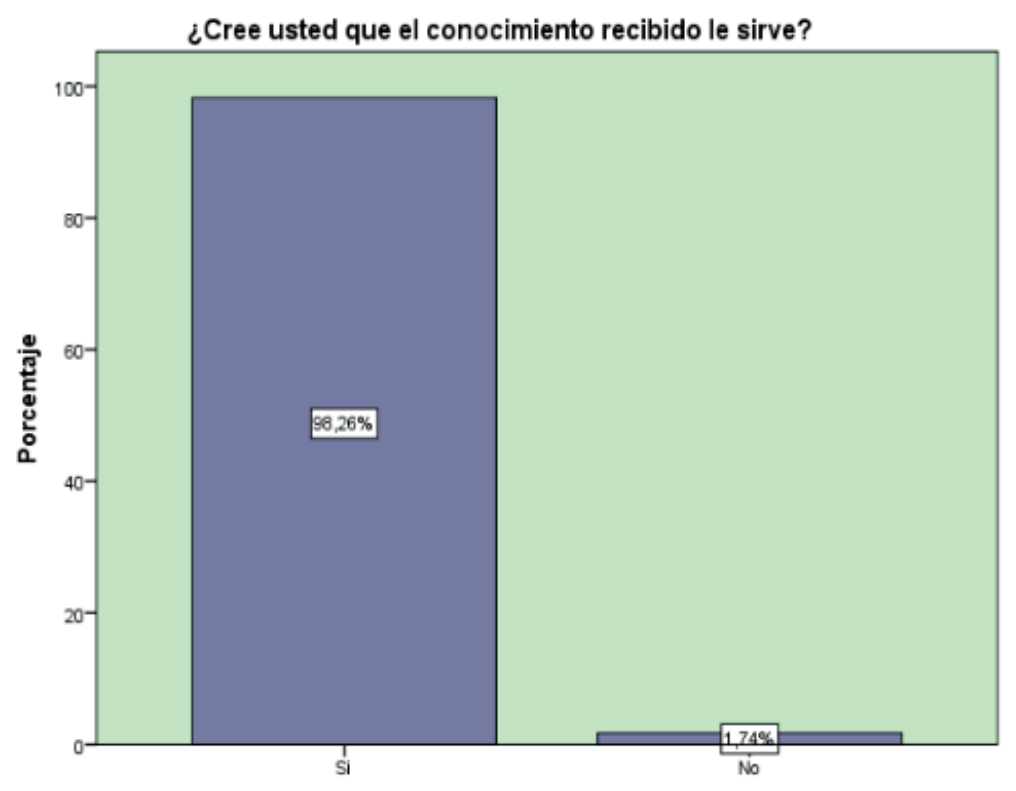




\section{Resultado}

Obtuvimos que un $90.26 \%$ de mujeres considera que sí les sirve en la aplicación de sus negocios, y el $1.76 \%$ manifestó que no. En otras palabras, la mayoría de mujeres pudo poner en práctica los conocimientos adquiridos, esto se da debido a que como se analizó en la pregunta anterior, el tipo de negocio y las materias que se impartieron se relacionaban a la actividad que realizaban y con ello en el momento de cumplir con sus jornadas de trabajo diario y así establecer el vínculo con los aprendizajes adquiridos. El 1,74\% de las mujeres no pudo establecer dicha relación.

\section{Variable: cumplimiento de expectativas}

El cumplimiento de las expectativas de las participantes del proyecto nos permite determinar si se pudo cumplir con el objetivo establecido al inicio del proyecto. Esta variable tiene relación directa con el tipo de actividades, metodologías y conocimientos que se impartan, es decir, que se mide de acuerdo a otras variables tomadas en el proyecto como la edad y tipo de negocio.

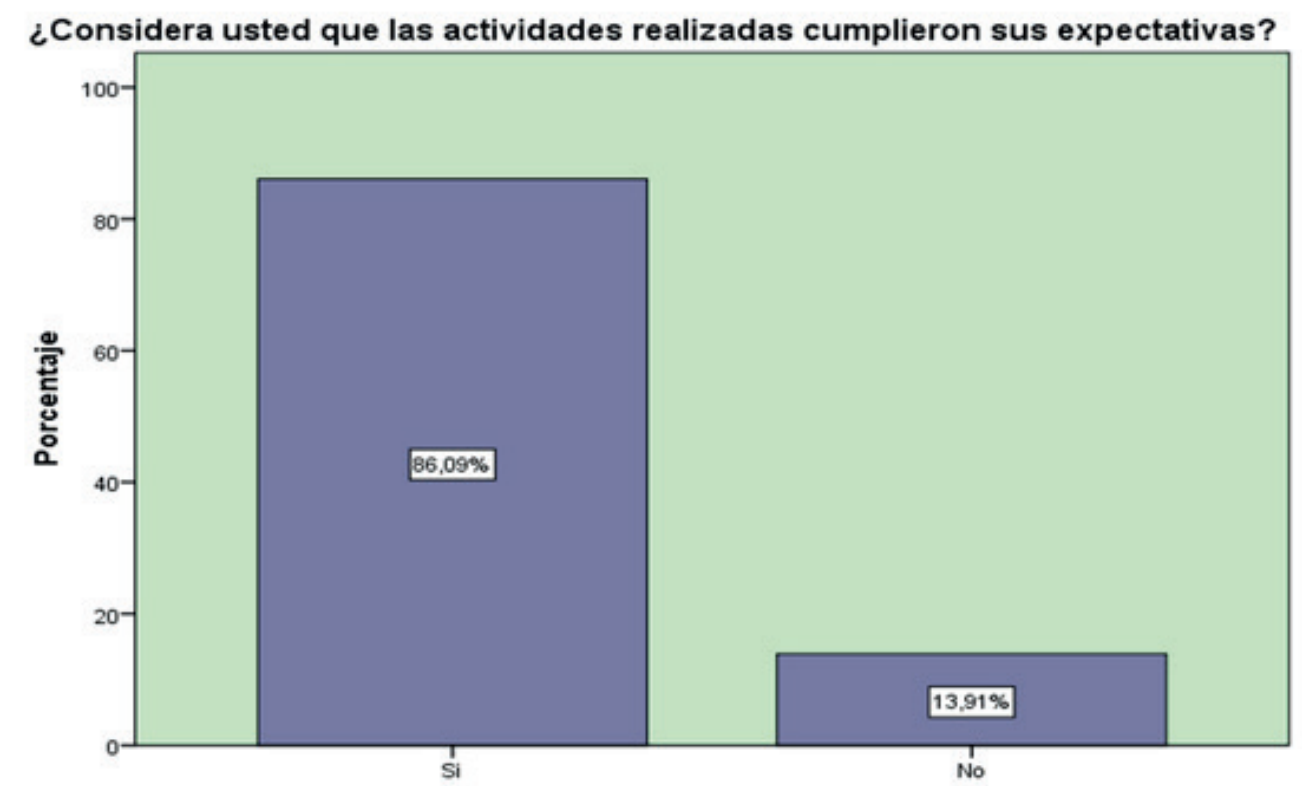

\section{Resultado}

El 86,09\% de los participantes indicó que el proyecto cumplió con sus expectativas y el 13,91\% no cumplió. Este resultado se obtuvo debido a que algunas variables, como la edad, y la metodología que se utilizó en el momento de impartir la capacitación, al igual que los conocimientos que se compartieron y el tipo de negocio, son factores que intervinieron directamente con el logro de dichas expectativas.

\section{Cruce de variables}

\section{Variables: edad - tipo de negocio}

Es importante analizar este tipo de variables ya que así nos permite determinar qué tipo de negocio es el que las mujeres desarrollan tomando en cuenta su edad, y así determinar qué tipo de capacitaciones es la más se necesita en la zona. 


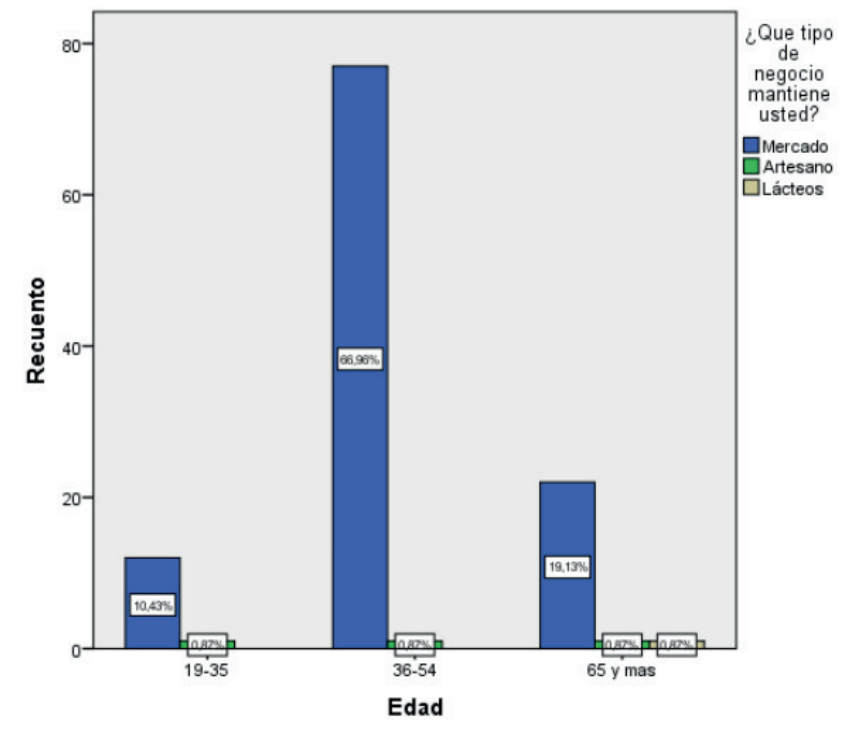

\section{Resultado}

El $66.96 \%$ de las mujeres entre los 36 y 54 años tiene un negocio de mercado, mientras que el negocio de lácteos solo lo manejan las mujeres de 65 años en adelante. En el mismo porcentaje están las artesanas de todas las edades. Podemos determinar que la mayor parte de mujeres llevan a cabo el tipo de negocio de mercado sin importar la edad, pero de lácteos solo las mujeres que llevan una tradición de comercializar este producto.

\section{Variables: tipo de negocio/conocimientos}

Estas variables se relacionaron debido a que para la capacitación brindada se analizó de qué materias les sería de mayor utilidad a las mujeres dependiendo de la actividad que realicen. Por este motivo se compara para determinar en qué porcentaje les fue de utilidad a las mujeres.

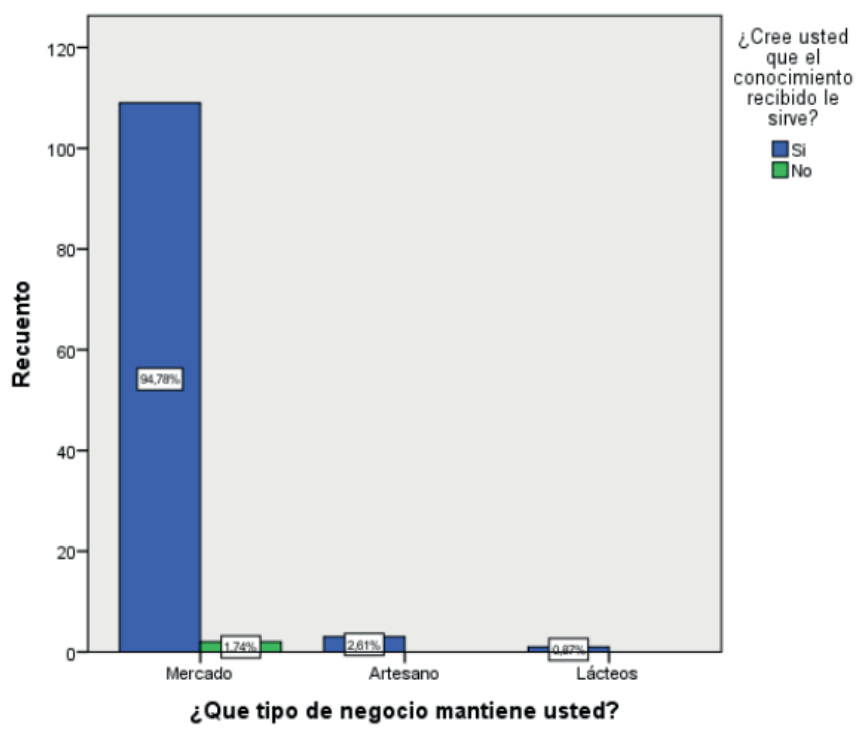




\section{Resultado}

El $94,78 \%$ de las mujeres que realizan la actividad de mercado manifestó que sí les sirvieron los conocimientos recibidos, y en el caso de las que realizan la actividad de artesanías y venta de lácteos su respuesta fue positiva. Es decir, que las instrucciones brindadas fueron las correctas, por lo tanto, estuvieron relacionadas a las actividades que desarrollaban.

\section{Variables: tipo de negocio/capacitación}

Se analizaron estas variables debido a que se quiso determinar en qué porcentaje y dependiendo del tipo de negocio desarrollaban. Las mujeres calificaron a la actividad realizada, para que, en posteriores aplicaciones del programa, poder realizar mejoras y contribuir al proyecto.

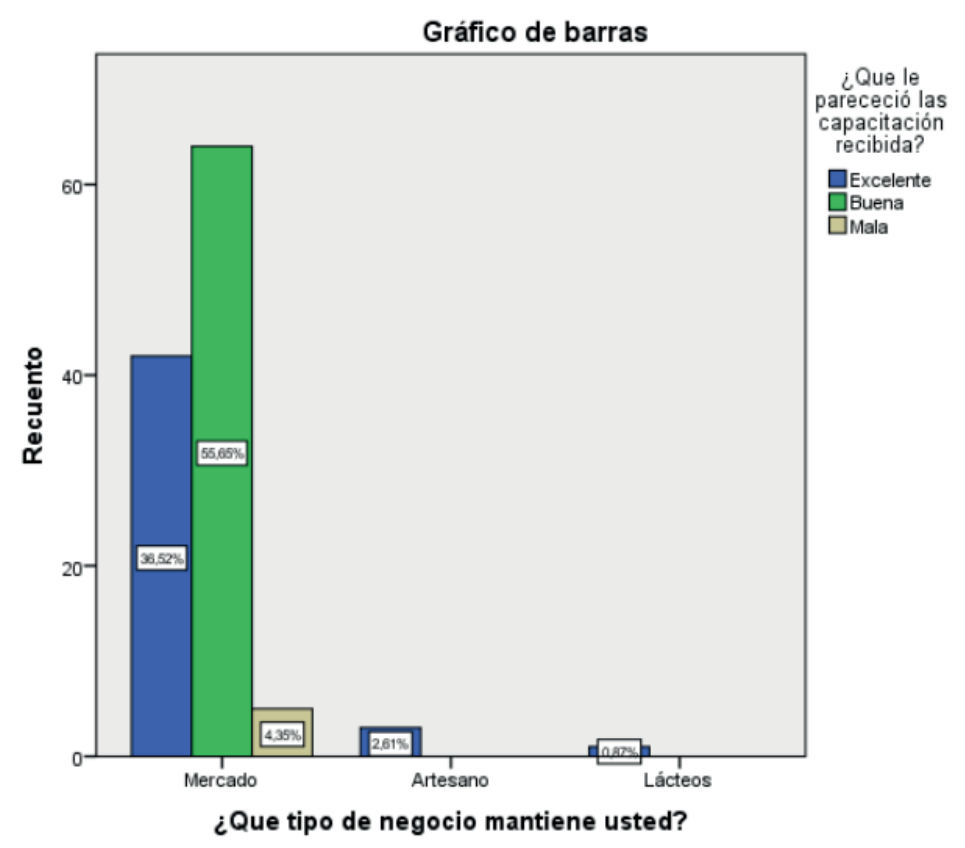

\section{Resultado}

Se pudo determinar que la mayor parte de las mujeres emprendedoras de la zona calificaron entre excelente y buena a las capacitaciones brindadas; es decir, que sí contribuyó a sus actividades, por lo que sería recomendable continuar con este proyecto en otras zonas que necesiten este aporte.

\section{Indicadores}

\section{Indicador de impácto del proyecto}

La medición del impacto de los proyectos se puede realizar mediante la verificación de la mejora en la calidad de vida, a lo que los participantes respondieron que sí en un 99,13\% y únicamente el 0,87\% indicó que no. A partir de este dato se puede construir el indicador de impacto del proyecto de vinculación con la sociedad. 


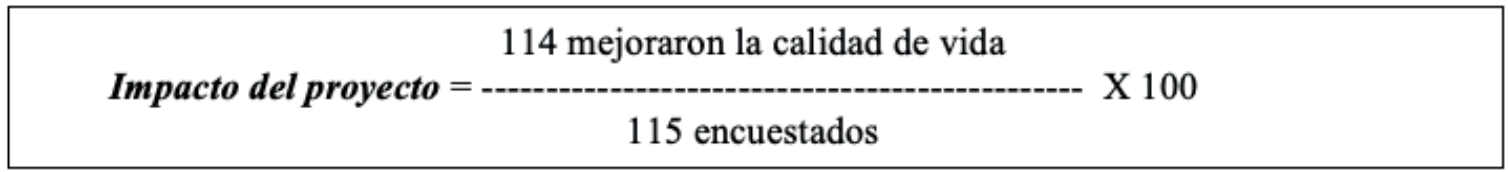

Impacto del proyecto $=99 \%$ en la mejora de calidad de vida

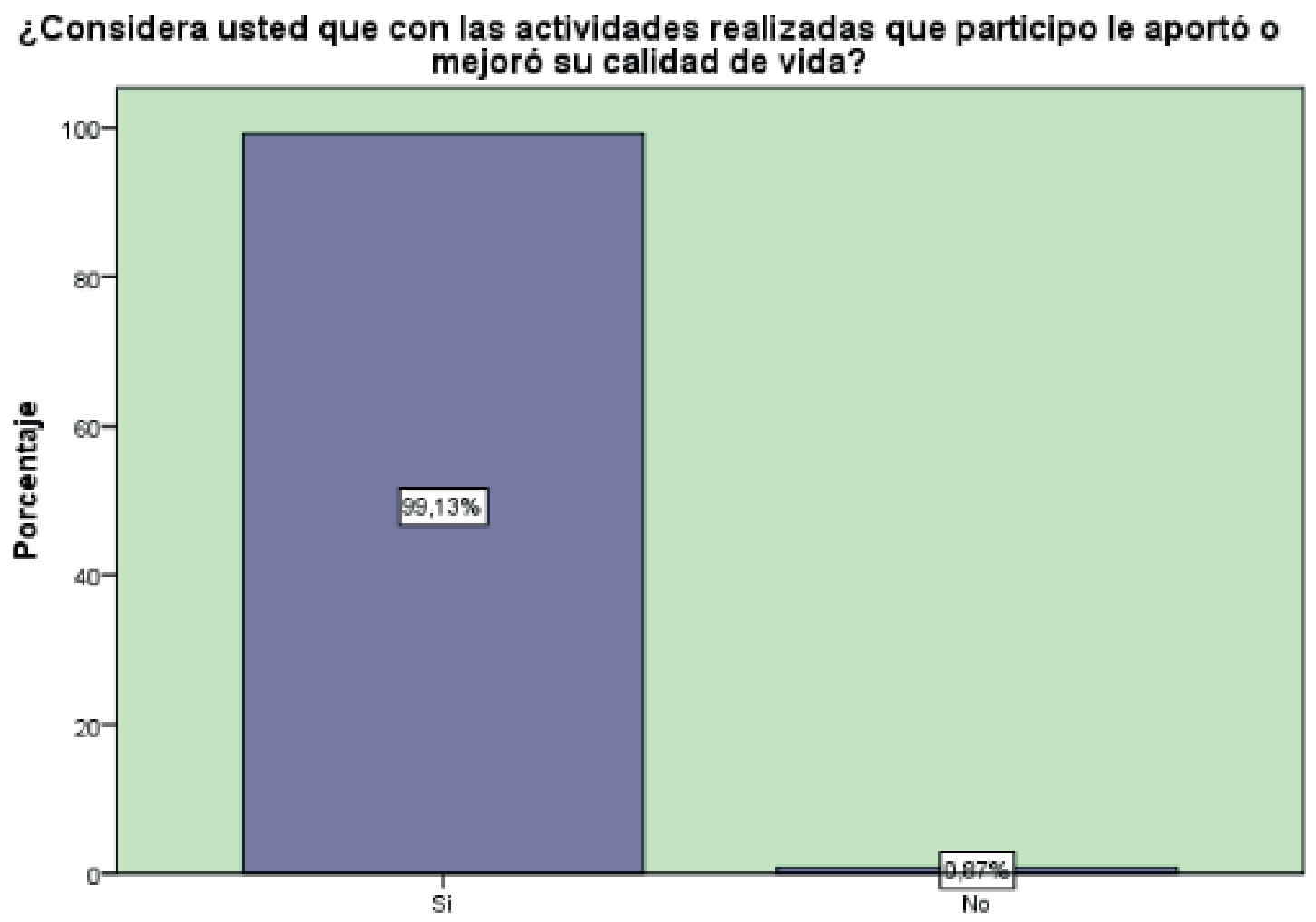

La calidad de vida es en donde se analiza el bienestar físico, material, social, emocional y el factor de desarrollo en el que contribuyó este proyecto facilitando el acceso al aprendizaje y asi permitir que las mujeres puedan contribuir y ser productivas en su sector.

\section{Indicador de cumplimiento del objetivo}

Los participantes indicaron en un 96,65\% que el proyecto sí cumplió con el objetivo y un 4,35\% que no. En la metodología del BID, en el indicador del cumplimiento del objetivo del proyecto, se obtuvo lo siguiente:

\begin{tabular}{|c|c|}
\hline \multirow{2}{*}{ Cumplimiento del objetivo } & $\begin{array}{c}110 \text { cumplió con el objetivo } \\
=\end{array}$ \\
\hline$-0-115$ encuestados
\end{tabular}

Cumplimiento del objetivo $=95,65 \%$ 


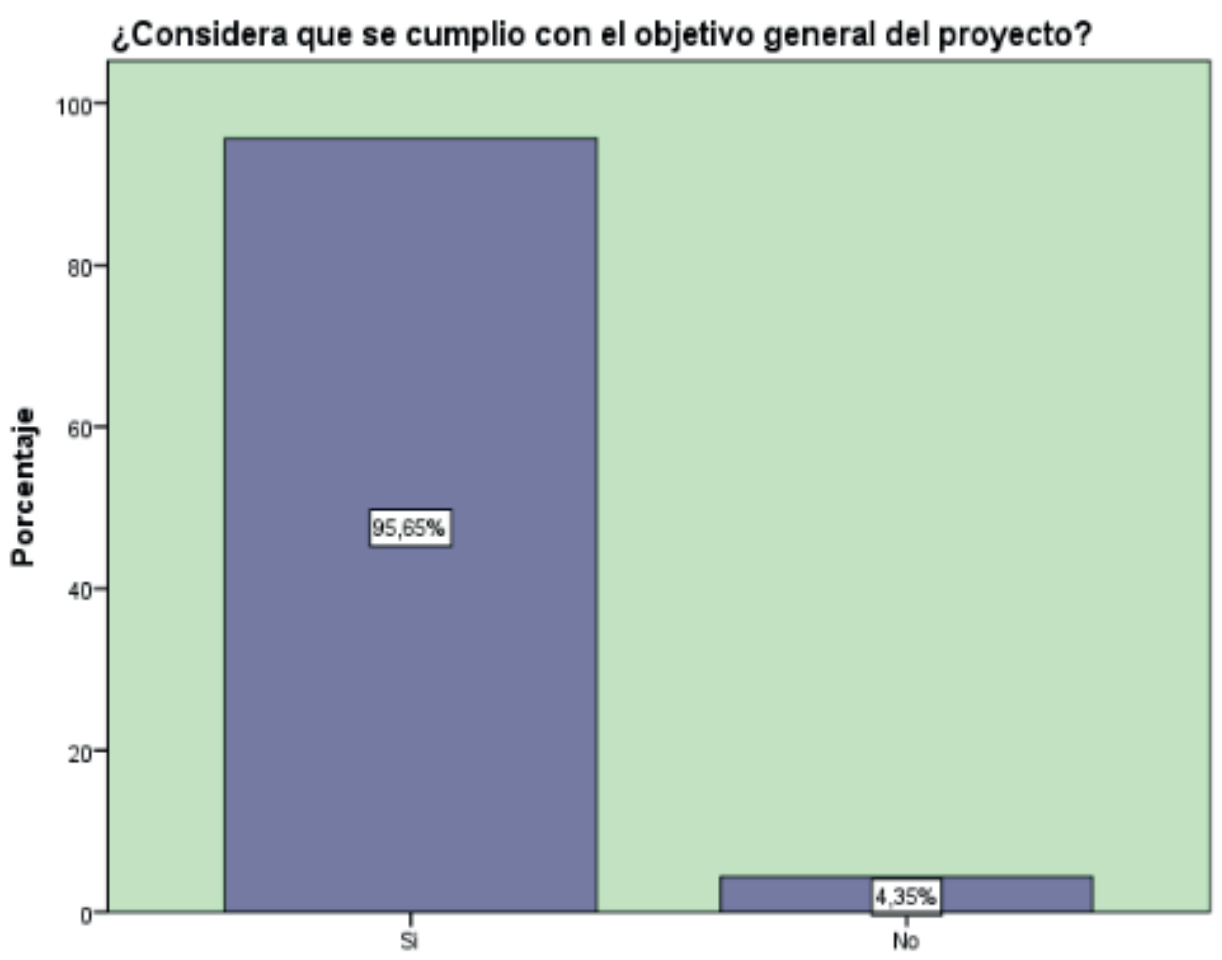

Elaborado por: Los autores

Para poder medir el cumplimiento del objetivo de este proyecto, se tomaron en cuenta diferentes factores que intervinieron en el desarrollo y fueron de importancia para el impacto que se alcanzó en el programa de inclusión de las mujeres emprendedoras.

\section{Indicador de satisfacción}

Se obtuvo que un $40 \%$ de los participantes del proyecto consideraran que fue excelente, un $55,65 \%$ que fue bueno y solo el 4,35\% que fue malo. En la metodología del BID, en el indicador del porcentaje de participantes que consideran satisfactorio al proyecto, se obtuvo lo siguiente:

\begin{tabular}{|c|c|}
\hline Porcentaje de satisfacción & 46 excelente +64 bueno \\
& 115 encuestados \\
\hline
\end{tabular}

Porcentaje de satisfacción $=95,65 \%$ 


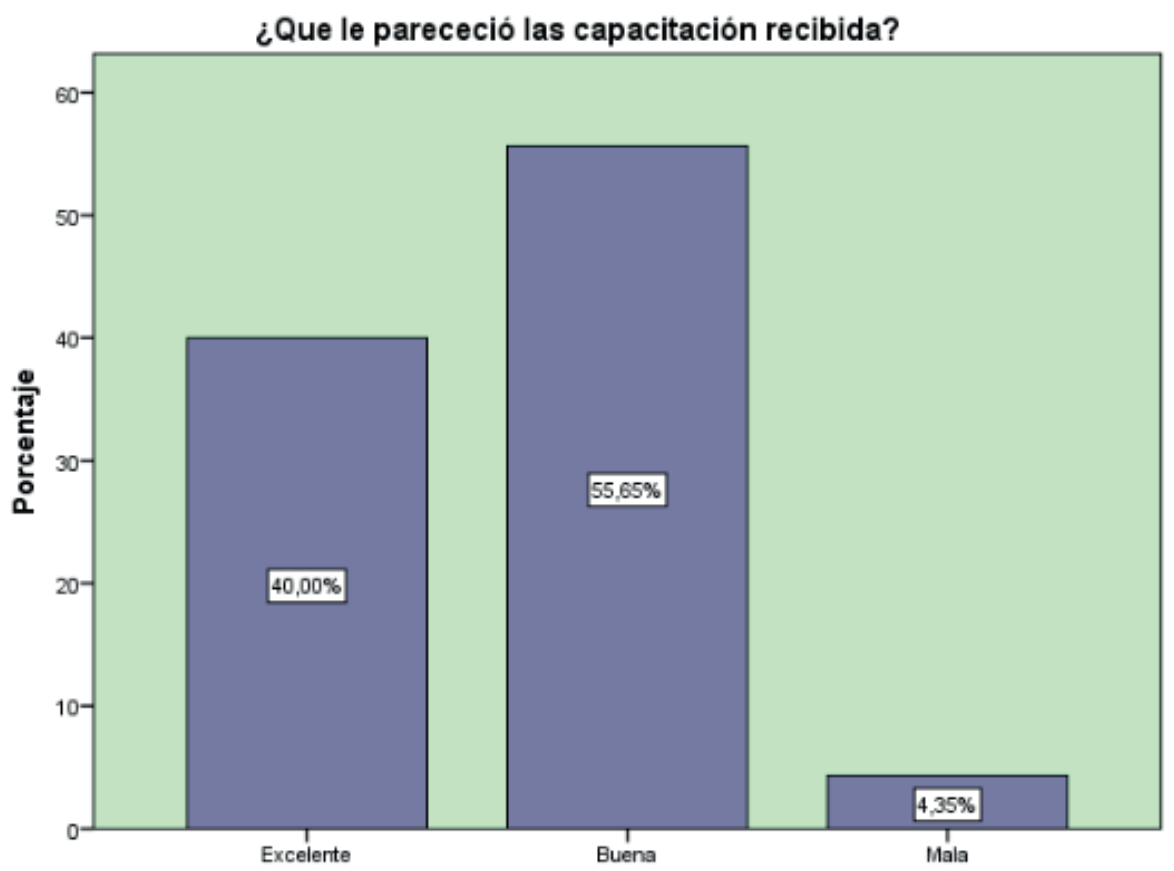

Elaborado por: Los autores

El tipo de capacitación que se brindó a las mujeres, permitió determinar en qué manera se llegó a las participantes y que así ellas pudieran calificar el grado de satisfacción que alcanzaron mediante los conocimientos que esperaban y lograron obtener.

\section{Discusión}

Una parte fundamental de la academia es que exista una vinculación entre los conocimientos y la sociedad, a fin de beneficiar directamente a los estudiantes que ponen en práctica dichos conocimientos y a la comunidad. En la actualidad, se está poniendo énfasis en la aplicación de dichos proyectos en las universidades del Ecuador.

Con frecuencia posterior a la aplicación de los proyectos se deja de lado la evaluación final del impacto social. Sin dicha evaluación, resulta imposible tener un parámetro que permita establecer la necesidad de continuar con los proyectos, realizar réplicas, o simplemente descartarlos. Median- te el presente estudio se ha obtenido un modelo de evaluación en función del BID que puede ser aplicable a otros proyectos de vinculación de la Facultad de Ciencias Administrativas, otras Facultades de la Universidad u otras universidades.

El presente proyecto alcanzó un impacto del $99 \%$ en la mejora de la calidad de vida según los resultados de los participantes, por cuanto es fundamental el fortalecimiento nuevos proyectos de vinculación con la sociedad en un trabajo conjunto entre la academia y el aporte a la comunidad con conocimientos para el desarrollo.

En el indicador del cumplimiento del objetivo del proyecto de vinculación, se obtuvo un $95,65 \%$, lo cual indica que se ha cumplido el objetivo planteado que fue "Fortalecer las habilidades empresariales de las mujeres del Cantón Cayambe a través de la capacitación en educación contable y financiera". Adicionalmente, los responsables deben pensar en la posibilidad de replicar el modelo. 
El indicador de porcentaje de satisfacción fue exactamente igual al cumplimiento del objetivo con un 95,65\%, lo que indica una altísima satisfacción por parte de los participantes.

Se obtuvo que al 86,09\% les gustaría recibir nuevas capacitaciones, y con un porcentaje de impacto del 99\%, cumplimiento del objetivo y satisfacción del objetivo del $95,65 \%$. Es fundamental mantener el proyecto de vinculación objeto de estudio, fortalecerlo y ampliarlo para otras zonas que requieran capacitaciones en temas administrativos, contables y financieros para un mejor desempeño de las comunidades.

\section{Conclusiones}

- Mediante el estudio se ha demostrado que el proyecto de vinculación con la colectividad de la Facultad de Ciencias Administrativas determinado: Programa de Inclusión Productiva y Educación Contable Financiera para Mujeres Microemprendedoras en el Cantón Cayambe, realizado durante el período 2016, tiene un impacto positivo del $99 \%$ en cuanto a que mejoró la calidad de vida de los participantes. De esta manera, la hipótesis se cumple con respecto al mejoramiento de la calidad de vida de los participantes del proyecto de vinculación con la sociedad.

- Se ha verificado que el proyecto de vinculación cumplió con el objetivo en un $95,65 \%$, el cual fue fortalecer las habilidades empresariales de las mujeres del cantón Cayambe a través de la capacitación en educación contable y financiera, permitiendo el desarrollo de los negocios que emprendieron varias mujeres de la zona. De esta manera, la hipótesis se cumple en cuanto al objetivo principal del proyecto de vinculación con la sociedad.

- Los participantes calificaron al proyecto con un $40 \%$ de excelente, $55,65 \%$ de bueno y $4,35 \%$ de malo, motivo por el que el porcentaje de satisfacción fue de $95,65 \%$, estableciendo que las mujeres emprendedoras sí pudieron poner en práctica en sus negocios lo recibido en el programa.

- El proyecto de vinculación con la sociedad contribuyó a mejorar conocimientos de contabilidad, tributación, manejo de costos y atención al cliente. Conocimientos de suma importancia en el desarrollo de emprendimientos.

\section{Bibliografía}

Angelelli P. y Prats J. (2005). Fomento de la actividad emprendedora en América Latina y el Caribe. Washington D.C. : BID.

Araque, Y. V. (2016). La Responsabilidad Social Universitaria: emprendimiento sostenible como impacto de intervención en comunidades vulnerables. Revista EAN, 20.

Arencibia, A. S. (2018). Metodología para evaluar el impacto en el desarrollo local del programa de maestría en contabilidad gerencial de la Universidad de Holguín. Revista de la Asociación de Inspectores de Educación de España , 27.

Barragán J.osé Barragán Codina, M. B. (2017). Impacto que tiene la Inversión en Educación Superior en el. International Journal of Good Conscience, 11.

Berdegué, J. A. (2015). Inclusión productiva rural. Estrategia de monitoreo y evaluación de los componentes de inclusión productiva de PROSPERA, 32.

Cañón Rodríguez, R. (2016). bbrecha digital: impacto en el desarrollo social y. tendencias pedagógicas , 19.

García, Á. (2017). Llas competencias en la formación del profesionalcontable: una revisión 
de las posturas institucionales y educativas . Revista Facultad de Ciencias Económicas: Investigación y Reflexión, , 83-1'3.

Herrandiz, A. C. (2016). Apuntes sobre la superación profesional en instituciones de la Educación Superior. Educación y Sociedad, 14

Lasio, V. (2016). Global Entrepeneurship Monitor. Guayaquil: Espol.

Lundy M.ark Lundy, R. I.-P. (2015). Un instrumento efectivo de inclusión productiva, versátil ante diferentes condiciones de vulnerabilidad y contextos regionales. POLITICA EN SINTESIS , 8.

Ortega Daniel D. Ortega y Kamiya Marco Kamiya. (2013). Emprendimientos en América Lati- na: Desde la subsistencia hacia la transformación productiva. Bogota: CAF.

Robayo, G. (2009). El emprendimiento y la educación no siempre van en la misma dirección. Polémika.USFQ .

Saavedra G., M. L. (2015). La investigación contable en Latinoamérica. Red de Revistas Científicas de América Latina, el Caribe, España y Portugal , 24.

Tomaselli, A. (2018). La educación técnica en el Ecuador. Ppolíticas sociales, 49.

La presente investigación fue realizada con la colaboración de la Coordinación de Investigación Formativa (CIF) y proyectos semilla de la Universidad Central del Ecuador. 